\title{
A CONSTRUCTION OF A MAXIMAL MONOTONE EXTENSION OF A MONOTONE MAP*
}

\author{
Jean-Pierre Crouzeix ${ }^{1}$, Eladio Ocaña Anaya ${ }^{2}$ and Wilfredo Sosa ${ }^{2}$
}

\begin{abstract}
A proof based on the axiom of choice shows that any monotone map has maximal monotone extensions but this proof is not constructive. In this paper, we give a construction of such an extension. The process is based on some density properties of (maximal) monotone maps given before.

Résumé. Afin de montrer qu'une multi-application monotone possède une extension maximale monotone, on utilise en général l'axiome du choix ou, ce qui est équivalent, le lemme de Zorn. Ce procédé est alors non constructif. Nous proposons ici une démonstration constructive de cette extension.
\end{abstract}

\section{INTRODUCTION}

The history of (maximal) monotone multi-valued maps started in the sixties with the pioneering works of Minty [8-10], see also Kachurovskii [4,5]. A classical survey on the monotonicity of maps defined on Hilbert spaces is the book by Brezis [2], see also Phelps [11]. A more recent survey is the book by Rockafellar and Wets [16] where the readers could find the most important results and a comprehensive bibliography.

In the present paper we consider multi-valued maps defined on finite dimensional spaces. As in [7] we tackle the monotonicity via the graphs of the maps instead of the maps themselves. We think that, in the same manner that the real essence of the convexity of a function is more present in the convexity of its epigraph than in the analytic definition, the true nature of monotonicity is more in the graphs than in the maps.

Any monotone map has a maximal monotone extension. The classical proof uses the axiom of choice (or the Zorn's lemma) and thereby is not constructive. In the last section, we show how to build a maximal monotone extension of a monotone map, and thereby we obtain a constructive proof of the existence. In the previous sections, after a short background on (maximal) monotonicity, we establish some basic properties of maximal monotone extensions used in the construction.

In the paper $X$ is a finite dimensional space and $X^{*}$ is its dual space. Of course $X^{*}$ can be identified with $X$, nevertheless we prefer to distinguish $X$ and $X^{*}$ in order to set in evidence their two different roles.

Given a closed convex set $C$ of $X$ and $x \in C$, we denote by $T_{C}(x)$ and $N_{C}(x)$ the tangent cone and the normal cone to $C$ at $x$ respectively.

\footnotetext{
* The research of the second author has been done when preparing his Ph.D. in Université Blaise Pascal, France.

1 LIMOS, Université Blaise Pascal, 63170 Aubière, France.

2 Instituto de Matematica y Ciencias Afines, Jr Ancash 536, Cercado de Lima, Lima, Perú.
} 


\section{Definitions And Basic Results}

A set $F \subset X \times X^{*}$ is said to be monotone if

$$
\left\langle x^{*}-y^{*}, x-y\right\rangle \geq 0 \text { for all }\left(x, x^{*}\right),\left(y, y^{*}\right) \in F
$$

and it is said maximal monotone if for any monotone subset $G$ of $X \times X^{*}$ containing $F$, we have $F=G$.

Let us associate with $F$ the set $\widetilde{F} \subset X \times X^{*}$ defined by

$$
\widetilde{F}:=\left\{\left(x, x^{*}\right) \in X \times X^{*}:\left\langle x^{*}-y^{*}, x-y\right\rangle \geq 0 \text { for all }\left(y, y^{*}\right) \in F\right\} .
$$

By construction, $\widetilde{F}$ is closed since it is an intersection of closed subsets.

The following properties are direct consequences of the definitions.

Proposition 1.1. Assume that $F$ and $G$ are two subsets of $X \times X^{*}$.

a) If $F \subset G$ then $\widetilde{G} \subset \widetilde{F}$.

b) $F$ is monotone if and only if $F \subset \widetilde{F}$.

c) If $F \subset G$ and $G$ is monotone then $F$ is monotone and $\widetilde{G} \subset \widetilde{F}$.

d) $F$ is maximal monotone if and only if $F=\widetilde{F}$.

e) $F$ is monotone if and only if $\mathrm{cl}(\mathrm{F})$ is monotone.

f) If $F$ is maximal monotone, then it is closed.

In particular, b) and c) say that if $F$ is monotone then $\widetilde{F}$ contains all monotone extensions of $F$ and, in particular, all the maximal monotone extensions.

In association with $G \subset X \times X^{*}$, we introduce the multi-valued maps $\Gamma$ and $\Gamma^{-1}$ defined respectively on $X$ and $X^{*}$ by

$$
\Gamma(x):=\left\{x^{*} \in X^{*}:\left(x, x^{*}\right) \in G\right\} \text { and } \Gamma^{-1}\left(x^{*}\right):=\left\{x \in X:\left(x, x^{*}\right) \in G\right\} .
$$

Thus $G$ can be considered, up to a permutation of variables, as the graph of both maps $\Gamma$ and $\Gamma^{-1}$. If $G$ is (maximal) monotone, then $\Gamma$ and $\Gamma^{-1}$ are said (maximal) monotone.

In connection with the construction of $\widetilde{G}$ from $G$, we associate with $\Gamma$ and $\Gamma^{-1}$ the maps

$$
\widetilde{\Gamma}(x):=\left\{x^{*} \in X^{*}:\left(x, x^{*}\right) \in \widetilde{G}\right\} \text { and } \widetilde{\Gamma^{-1}}\left(x^{*}\right):=\left\{x \in X:\left(x, x^{*}\right) \in \widetilde{G}\right\} .
$$

The domains of $\Gamma$ and $\Gamma^{-1}$ are the projections of $G$ on $X$ and $X^{*}$,

$$
\begin{aligned}
\operatorname{dom}(\Gamma) & :=\{x: \Gamma(x) \neq \emptyset\}=\operatorname{proj}_{\mathrm{x}}(\mathrm{G}), \\
\operatorname{dom}\left(\Gamma^{-1}\right) & :=\left\{x^{*}: \Gamma^{-1}\left(x^{*}\right) \neq \emptyset\right\}=\operatorname{proj}_{\mathrm{X}}^{*}(\mathrm{G}) .
\end{aligned}
$$

Clearly, $\widetilde{\Gamma}^{-1}=\widetilde{\Gamma^{-1}}$ and, thanks to Proposition 1.1, the equality $\widetilde{\Gamma}=\Gamma$ (equivalently $\widetilde{\Gamma}^{-1}=\Gamma^{-1}$ ) holds if and only if the multi-valued map $\Gamma$ is maximal monotone.

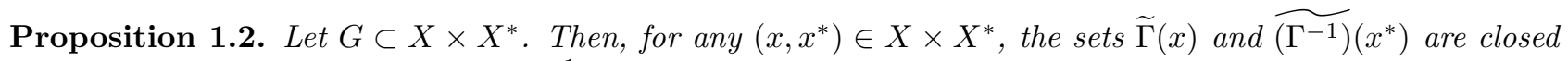
and convex. In particular, $\Gamma(x)$ and $\Gamma^{-1}\left(x^{*}\right)$ are closed and convex when $G$ is maximal monotone.

Proof. By definition,

and

$$
\widetilde{\Gamma}(x)=\bigcap_{\left(y, y^{*}\right) \in \widetilde{G}}\left\{x^{*}:\left\langle x^{*}-y^{*}, x-y\right\rangle \geq 0\right\}
$$

$$
\widetilde{\left(\Gamma^{-1}\right)}\left(x^{*}\right)=\bigcap_{\left(y, y^{*}\right) \in \widetilde{G}}\left\{x:\left\langle x^{*}-y^{*}, x-y\right\rangle \geq 0\right\} .
$$

These sets are closed and convex as intersections of closed half-spaces. 
Given $A \subset X$, we denote by co $(\mathrm{A})$ and $\overline{\mathrm{co}}(\mathrm{A})$ the convex hull and the closed convex hull of $A$. Next, given a multi-valued map $\Gamma: X \longrightarrow X^{*}$, we define the multi-valued maps co $\Gamma$ and $\overline{\operatorname{co}} \Gamma$ by $(\operatorname{co} \Gamma)(x)=\operatorname{co}(\Gamma(x))$ and $(\overline{\mathrm{co}} \Gamma)(x)=\overline{\mathrm{co}}(\Gamma(x))$ for all $x \in X$. The following result is a direct consequence of Proposition 1.1.

Proposition 1.3. If one of the maps $\Gamma, \operatorname{co} \Gamma, \overline{\mathrm{co}} \Gamma$ is monotone, so are the other ones.

Also Proposition 1.3 implies that if $G$ is maximal monotone, then $\Gamma(x)$ and $\Gamma^{-1}\left(x^{*}\right)$ are closed and convex for all $x \in X, x^{*} \in X^{*}$.

\section{Some properties of $\widetilde{\Gamma}$}

In complement with the last section, we give less immediate results on $\widetilde{\Gamma}$. The first of them is well known when $\widetilde{\Gamma}=\Gamma$, i.e., when $\Gamma$ is maximal monotone. Its proof is quite easy.

Proposition 2.1. Let $\Gamma: X \longrightarrow X^{*}$ be a monotone multi-valued map and $C=\overline{\operatorname{co}} \operatorname{dom}(\Gamma)$. Assume that $\bar{x} \in \operatorname{dom}(\widetilde{\Gamma}) \cap \mathrm{C}$. Then, $N_{C}(\bar{x})$ coincides with the recession cone of $\widetilde{\Gamma}(\bar{x})$, i.e.,

$$
N_{C}(\bar{x})=(\widetilde{\Gamma}(\bar{x}))_{\infty}
$$

It follows that

$$
\widetilde{\Gamma}(x)=\widetilde{\Gamma}(x)+N_{C}(x) \text { for all } x \in C .
$$

Proof. By definition, $w^{*} \in N_{C}(\bar{x})$ if and only if

$$
\left\langle x^{*}+t w^{*}-y^{*}, \bar{x}-y\right\rangle \geq 0 \text { for all }\left(y, y^{*}\right) \in \operatorname{graph}(\Gamma), \mathrm{t} \geq 0 \text { and } x^{*} \in \widetilde{\Gamma}(\bar{x}) .
$$

Thus, $N_{C}(\bar{x})$ consists of the vectors $w^{*}$ such that

$$
x^{*}+t w^{*} \in \widetilde{\Gamma}(\bar{x}) \text { for all } x^{*} \in \widetilde{\Gamma}(\bar{x}) \text { and } t \geq 0 .
$$

This condition means that $N_{C}(\bar{x})$ coincides with the recession cone of the closed convex set $\widetilde{\Gamma}(x)$.

For the forthcoming results, we need the following technical lemma.

Lemma 2.2. Let $D=\operatorname{co}\left\{x_{0}, x_{1}, \cdots, x_{\mathrm{n}}\right\}$ be an $n$-dimensional simplex of $\mathbb{R}^{n}, V$ be a closed convex subset of int (D). Then for any $x \in V$ and any $c \in \mathbb{R}^{n}$ the following linear program

$$
\inf _{u}\left[\sum_{i=0}^{n} u_{i}: u_{i} \geq 0, \sum_{i=0}^{n} u_{i}\left(x_{i}-x\right)=c\right]
$$

is feasible and has a unique optimal solution. Denote it by $u(c, x)$. Then the function $(c, x) \mapsto u(c, x)$ is continuous on $X \times V$.

Proof. Because $x \in V \subset$ int (D), the $n$ vectors $\left(x_{i}-x\right), i=1,2, \cdots, n$ are linearly independent. Hence there exist uniquely defined $\gamma_{i}(x)>0, i=1,2, \cdots, n$, such that

$$
x-x_{0}=\sum_{i=1}^{n} \gamma_{i}(x)\left(x_{i}-x\right) .
$$

Also, there are uniquely defined $\lambda_{i}(c, x) \in \mathbb{R}, i=1,2, \cdots, n$ such that

$$
c=\sum_{i=1}^{n} \lambda_{i}(c, x)\left(x_{i}-x\right) .
$$


Furthermore the functions $\gamma_{i}$ and $\lambda_{i}$ are continuous on $V$ and $\mathbb{R}^{n} \times V$ respectively.

Thus, $u$ is feasible for $(l p l)$ if and only if

$$
u=\left(u_{0}, u_{1}, \cdots, u_{n}\right) \geq 0 \text { and } \lambda_{i}(c, x)=u_{i}-u_{0} \gamma_{i}(x), \quad i=1,2, \cdots, n
$$

or equivalently

$$
u_{0} \geq 0 \text { and } \lambda_{i}(c, x)+u_{0} \gamma_{i}(x)=u_{i} \geq 0, \quad i=1,2, \cdots, n .
$$

Then $(l p l)$ is reduced to the one variable linear program

$$
\inf _{u_{0}}\left[u_{0}\left(1+\sum_{i=1}^{n} \gamma_{i}(x)\right): u_{0} \geq 0, u_{0} \geq-\frac{\lambda_{i}(c, x)}{\gamma_{i}(x)}, i=1,2, \cdots, n\right]
$$

Since $1+\sum_{i=1}^{n} \gamma_{i}(x)>0,\left(l p l_{1}\right)$ has a unique optimal solution, this is also the case for $(l p l)$. The optimal solution of $(l p l)$ is

$$
\begin{aligned}
& u_{0}(c, x)=\max \left\{0, \max _{i}\left[-\frac{\lambda_{i}(c, x)}{\gamma_{i}(x)}: i=1,2, \cdots, n\right]\right\} \\
& u_{i}(c, x)=u_{0}(c, x) \gamma_{i}(x) \quad \text { for } i=1,2, \cdots, n .
\end{aligned}
$$

The continuity of the function $(c, x) \mapsto u(c, x)$ is a consequence of the continuity of the functions $\gamma_{i}$ and $\lambda_{i}$.

In the following results we assume that the interior of the convex hull of the domain of $\Gamma$ is not empty. These results will be generalized in the next section with the relative interior in place of the interior.

Theorem 2.3. Let $\Gamma: X \longrightarrow X^{*}$ be monotone. Denote by $D$ the interior of the convex hull of the domain of $\Gamma$. Then, for all $\bar{x} \in D$, there exists a compact set $K \subset X^{*}$ and a neighbourhood $V$ of $\bar{x}$ such that $\emptyset \neq \widetilde{\Gamma}(x) \subset K$ for all $x \in V$. It follows that $D$ is contained in the interior of the domain of $\widetilde{\Gamma}$.

Proof. i) Assume that $X=\mathbb{R}^{n}$. Denote by $G$ the graph of $\Gamma$. Since $\bar{x}$ belongs to $D$, there exist $\bar{t}_{i}>0$ and $\left(x_{i}, x_{i}^{*}\right) \in G$ for $i=0,1, \cdots, n$ such that the $n$ vectors $\left(x_{i}-x_{0}\right), i=1,2, \cdots, n$, are linearly independent and

$$
\bar{x}=\sum_{i=0}^{n} \bar{t}_{i} x_{i}, \quad 1=\sum_{i=0}^{n} \bar{t}_{i}
$$

Let some $\epsilon>0$ be such that $\epsilon<\bar{t}_{i}$ for $i=0,1, \cdots, n$. Let $V$ be defined by

$$
V=\left\{x=\sum_{i=0}^{n} t_{i} x_{i}: 1=\sum_{i=0}^{n} t_{i} \quad \text { and } \quad \epsilon \leq t_{i} \text { for all } i\right\}
$$

Then $V$ is a compact convex neighbourhood of $\bar{x}$ contained in $D$. Given $c \in \mathbb{R}^{n}$ and $x \in V$, let us define

$$
\alpha(c, x)=\sup _{x^{*}}\left[\left\langle c, x^{*}\right\rangle: x^{*} \in \widetilde{\Gamma}(x)\right]
$$

with, by convention, $\alpha(c, x)=-\infty$ if $\widetilde{\Gamma}(x)=\emptyset$. Then,

$$
-\infty \leq \alpha(c, x) \leq \beta(c, x)
$$

where

$$
\beta(c, x)=\sup _{x^{*}}\left[\left\langle c, x^{*}\right\rangle:\left\langle x^{*}, x_{i}-x\right\rangle \leq\left\langle x_{i}^{*}, x_{i}-x\right\rangle, i=0,1,2, \cdots, n\right] .
$$


The dual of the linear program $\left(P_{e}\right)$ is the problem

$$
\widetilde{\beta}(c, x)=\inf _{u}\left[\sum_{i=0}^{n} u_{i}\left\langle x_{i}^{*}, x_{i}-x\right\rangle: u_{i} \geq 0 \text { and } \sum_{i=0}^{n} u_{i}\left(x_{i}-x\right)=c\right] .
$$

The optimal solution $u(c, x)$ of problem $(l p l)$ in Lemma 2.2 is a feasible solution of $\left(D_{e}\right)$. Define

$$
\rho(c, x)=\sum_{i=0}^{n} u_{i}(c, x)\left\langle x_{i}^{*}, x_{i}-x\right\rangle
$$

Then, $\widetilde{\beta}(c, x) \leq \rho(c, x)$. On the other hand, since $\left(D_{e}\right)$ is feasible, there is no duality gap between the two problems $\left(P_{e}\right)$ and $\left(D_{e}\right)$ and therefore

$$
\beta(c, x)=\widetilde{\beta}(c, x) \leq \rho(c, x)
$$

Next, define

$$
M=\sup _{x, c}[\rho(c, x): x \in V,\|c\| \leq 1] \text { and } K=\left\{x^{*}:\left\|x^{*}\right\| \leq M\right\} .
$$

Since $V$ is compact and the function $u$ is continuous on $\mathbb{R}^{n} \times V, M$ is finite and $K$ is a compact convex set.

Then, for all $c$ with $\|c\| \leq 1$ and all $x \in V$, one has

$$
\alpha(c, x) \leq \beta(c, x) \leq \rho(c, x) \leq M
$$

Define the quantities

$$
\begin{aligned}
& A(x)=\sup _{x^{*}}\left[\left\|x^{*}\right\|: x^{*} \in \widetilde{\Gamma}(x)\right] \\
& B(x)=\sup _{x^{*}}\left[\left\|x^{*}\right\|:\left\langle x^{*}-x_{i}^{*}, x_{i}-x\right\rangle \leq 0, \quad i=0,1, \cdots, n\right] .
\end{aligned}
$$

Then,

$$
A(x)=\sup _{c, x^{*}}\left[\left\langle c, x^{*}\right\rangle: x^{*} \in \widetilde{\Gamma}(x),\|c\| \leq 1\right]=\sup _{c}[\alpha(c, x):\|c\| \leq 1]
$$

and

$$
B(x)=\sup _{c}[\beta(c, x):\|c\| \leq 1] .
$$

The inequalities $A(x) \leq B(x) \leq M$ for all $x \in V$ deduced from (1) imply

$$
\widetilde{\Gamma}(x) \subset\left\{x^{*}:\left\langle x^{*}, x_{i}-x\right\rangle \leq\left\langle x_{i}^{*}, x_{i}-x\right\rangle, i=0,1, \cdots, n\right\} \subset K .
$$

ii) Assume for contradiction that $y \in V$ and $\tilde{\Gamma}(y)=\emptyset$. Then,

$$
\emptyset=\tilde{\Gamma}(y)=\bigcap_{\left(x, x^{*}\right) \in G}\left\{y^{*} \in \mathbb{R}^{n}:\left\langle y^{*}-x^{*}, y-x\right\rangle \geq 0\right\} \subset K .
$$

Since $K$ is compact, there exist a positive integer $q$ and points $\left(x_{j}, x_{j}^{*}\right) \in G$ for $j=n+1, n+2, \cdots, n+q$ such that

$$
\emptyset=\left[\bigcap_{j=n+1}^{j=n+q}\left\{y^{*}:\left\langle y^{*}-x_{j}^{*}, y-x_{j}\right\rangle \geq 0\right\}\right] \cap K
$$


Next, in view of (2),

$$
\emptyset=\bigcap_{j=0}^{j=n+q}\left\{y^{*}:\left\langle y^{*}-x_{j}^{*}, y-x_{j}\right\rangle \geq 0\right\} .
$$

Consider the $n \times(n+q+1)$ matrix $Q$ with columns $\left(y-x_{j}\right), j=0,1, \cdots n+q$, and the vector $a \in \mathbb{R}^{n+q+1}$ with components $a_{j}=\left\langle x_{j}^{*}, y-x_{j}\right\rangle$. Next, consider the pair of dual linear programs

$$
\begin{aligned}
& m_{1}=\inf _{y^{*}}\left[\left\langle 0, y^{*}\right\rangle: Q^{t} y^{*} \geq a\right], \\
& m_{2}=\sup _{u}[\langle a, u\rangle: u \geq 0, Q u=0] .
\end{aligned}
$$

The second problem is feasible and therefore $m_{1}=m_{2}$. Next, (3) is equivalent to $m_{1}=+\infty$ and therefore to

$$
\exists u \in \mathbb{R}^{n+q+1} \text { such that } u \geq 0, Q u=0 \text { and }\langle a, u\rangle>0 .
$$

Without loss of generality, we assume that $\sum u_{i}=1$. Then $Q u=0$ implies $y=\sum u_{i} x_{i}$ and

$$
\begin{aligned}
\langle a, u\rangle & =\sum_{j=0}^{n+q} u_{j}\left\langle x_{j}^{*}, \sum_{i=0}^{n+q} u_{i} x_{i}-x_{j}\right\rangle, \\
\langle a, u\rangle & =\sum_{j=0}^{n+q}\left\langle u_{j} x_{j}^{*}, \sum_{i=0}^{n+q} u_{i}\left(x_{i}-x_{j}\right)\right\rangle, \\
\langle a, u\rangle & =-\sum_{i, j=0}^{n+q} u_{i} u_{j}\left\langle x_{i}^{*}-x_{j}^{*}, x_{i}-x_{j}\right\rangle .
\end{aligned}
$$

Because the $\left(x_{i}, x_{i}^{*}\right)^{\prime} s$ belong to the graph of $\Gamma$ which is monotone, one has $\left\langle x_{i}^{*}-x_{j}^{*}, x_{i}-x_{j}\right\rangle \geq 0$ for all $i, j$, in contradiction with $u \geq 0$ and $\langle a, u\rangle>0$.

Let us apply this theorem to the particular case where $\Gamma$ is maximal monotone. Then $\widetilde{\Gamma}=\Gamma$ and we recover the fundamental result which says that $\Gamma(x)$ is locally bounded and nonempty on the interior of the domain of $\Gamma$. The next theorem also generalizes to monotone maps a result known for maximal monotone maps.

Theorem 2.4. Let $\Gamma: X \longrightarrow X^{*}$ be monotone. Assume that the interior of the convex hull of its domain is nonempty. Denote by $\widetilde{D}$ the domain of $\widetilde{\Gamma}$. Then the sets $\operatorname{int}(\widetilde{\mathrm{D}})$ and $\operatorname{cl}(\widetilde{\mathrm{D}})$ are convex. $\operatorname{Moreover}, \operatorname{cl}(\widetilde{\mathrm{D}})=$ $\operatorname{cl}(\operatorname{int}(\widetilde{\mathrm{D}}))$ and $\operatorname{int}(\widetilde{\mathrm{D}})=\operatorname{int}(\operatorname{cl}(\widetilde{\mathrm{D}}))$.

Proof. Fix some $a$ in the interior of the convex hull of the domain of $\Gamma$.

i) Let us prove that $\bar{x} \in \operatorname{cl}(\operatorname{int}(\widetilde{\mathrm{D}}))$ for any $\bar{x} \in \operatorname{cl}(\widetilde{\mathrm{D}})$. Consider a sequence $\left\{x_{k}\right\}_{k} \subset \widetilde{D}$ converging to $\bar{x}$. For each $k$, consider some $x_{k}^{*} \in \widetilde{\Gamma}\left(x_{k}\right)$. Next consider the map $\Gamma_{k}$ defined from its graph as follows

$$
\operatorname{graph}\left(\Gamma_{\mathrm{k}}\right)=\operatorname{graph}(\Gamma) \cup\left\{\left(x_{k}, x_{k}^{*}\right)\right\}
$$

Then $\Gamma_{k}$ is monotone, $\operatorname{dom}\left(\Gamma_{k}\right)=\operatorname{dom}(\Gamma) \cup\left\{x_{k}\right\}$ and $\widetilde{\Gamma}_{k} \subset \widetilde{\Gamma}$. Let $y_{k}=x_{k}+\frac{1}{k}\left(a-x_{k}\right)$, then by construction, $x_{k} \in \operatorname{dom}\left(\Gamma_{k}\right)$, the sequence $\left\{y_{k}\right\}_{k}$ converges to $\bar{x}$ and lies in the interior of the convex hull of the domain of $\Gamma_{k}$. It follows from Theorem 2.3 that $y_{k}$ belongs to the interior of the domain of $\widetilde{\Gamma}_{k}$, and therefore to the interior of the domain of $\widetilde{\Gamma}$.

ii) Let us prove that $\operatorname{int}(\widetilde{\mathrm{D}})$ is convex. Given $\bar{x}, \bar{y} \in \operatorname{int}(\widetilde{\mathrm{D}})$ and $\alpha \in(0,1)$, we must prove that $\bar{z}=$ $\alpha \bar{x}+(1-\alpha) \bar{y} \in \operatorname{int}(\widetilde{\mathrm{D}})$. There exists $\gamma>1$ such that $x=a+\gamma(\bar{x}-a)$ and $y=a+\gamma(\bar{y}-a)$ belong to int $(\widetilde{\mathrm{D}})$. Set $z=a+\gamma(\bar{z}-a)=\alpha x+(1-\alpha) y$. Let $x^{*} \in \widetilde{\Gamma}(x)$ and $y^{*} \in \widetilde{\Gamma}(y)$. We distinguish two cases. 
ii-a) First case: $\left\langle x^{*}-y^{*}, x-y\right\rangle \geq 0$. Take $\Sigma: X \longrightarrow X^{*}$ such that

$$
\operatorname{graph}(\Sigma)=\operatorname{graph}(\Gamma) \cup\left\{\left(x, x^{*}\right)\right\} \cup\left\{\left(y, y^{*}\right)\right\}
$$

By construction, $z$ belongs to the convex hull of $\operatorname{dom}(\Sigma), \Sigma$ is monotone and contains $\Gamma$. Hence, $\widetilde{\Sigma} \subset \widetilde{\Gamma}$. Since $a$ belongs to the interior of the convex hull of $\operatorname{dom}(\Sigma), \bar{z}=\gamma^{-1} z+\left(1-\gamma^{-1}\right) a$ belongs also to the interior. Then, Theorem 2.3 implies that $\bar{z}$ belongs to the interior of the domain of $\widetilde{\Sigma}$ and, thereby to the interior of the domain of $\widetilde{\Gamma}$.

ii-b) Second case: $\left\langle x^{*}-y^{*}, x-y\right\rangle<0$. We shall prove that $z^{*}=\alpha x^{*}+(1-\alpha) y^{*} \in \widetilde{\Gamma}(z)$, that is to say, $A=\left\langle z^{*}-w^{*}, z-w\right\rangle \geq 0$ for any $\left(w, w^{*}\right) \in \operatorname{graph}(\Gamma)$. Let us compute $A$.

$$
\begin{aligned}
A= & \left\langle\alpha\left(x^{*}-y^{*}\right)+\left(y^{*}-w^{*}\right),(1-\alpha)(y-x)+(x-w)\right\rangle \\
A= & \alpha(1-\alpha)\left\langle x^{*}-y^{*}, y-x\right\rangle+\alpha\left\langle x^{*}-w^{*}+w^{*}-y^{*}, x-w\right\rangle+ \\
& \cdots+(1-\alpha)\left\langle y^{*}-w^{*}, y-w+w-x\right\rangle+\left\langle y^{*}-w^{*}, x-w\right\rangle \\
A= & \alpha(1-\alpha)\left\langle x^{*}-y^{*}, y-x\right\rangle+\alpha\left\langle x^{*}-w^{*}, x-w\right\rangle+ \\
& \cdots+(1-\alpha)\left\langle y^{*}-w^{*}, y-w\right\rangle .
\end{aligned}
$$

The assumptions imply $A \geq 0$, hence $\left(z, z^{*}\right) \in \operatorname{graph}(\widetilde{\Gamma})$. Next, define $\Sigma: X \longrightarrow X^{*}$ by

$$
\operatorname{graph}(\Sigma)=\operatorname{graph}(\Gamma) \cup\left\{\left(z, z^{*}\right)\right\}
$$

Then, $\Sigma$ is monotone, contains $\Gamma$ and $\bar{z}$ belongs to the interior of the convex hull of its domain, and thereby to the interior of the domain of $\widetilde{\Sigma}$ which is contained in the interior of the domain of $\widetilde{\Gamma}$.

iii) The other claims of the theorem are direct consequences of i) and ii).

A monotone map $\Gamma$ may have different maximal monotone extensions. In the following results, we shall prove that the maximal monotone extension is uniquely defined when a density condition holds on the domain. Before, we introduce the following definition.

Definition 2.5. Given $\Gamma: X \longrightarrow X^{*}$ and $S \subset \operatorname{dom}(\Gamma)$, we define the map $\Gamma_{\mathrm{S}}: X \longrightarrow X^{*}$ from its graph

$$
\operatorname{graph}\left(\Gamma_{\mathrm{S}}\right)=\operatorname{cl}\left[\operatorname{graph}(\Gamma) \cap\left(\mathrm{S} \times \mathrm{X}^{*}\right)\right]
$$

It is clear that if $\Gamma$ is monotone, so are $\Gamma_{\mathrm{S}}$ and $\overline{\mathrm{co}}\left(\Gamma_{\mathrm{S}}\right)$. In the following results we denote by $C$ the closed convex hull of dom $(\Gamma)$ and we assume that the interior of $C$ is not empty. The first result considers points in the interior of $C$ and the next two results deal with points in the boundary.

Theorem 2.6. Let $\Gamma: X \longrightarrow X^{*}$ be monotone and $S \subset \operatorname{dom}(\Gamma)$. Let $V \subset C$ be open, convex and such that $\mathrm{cl}(\mathrm{V} \cap \mathrm{S})=\operatorname{cl}(\mathrm{V})$. Then, $\widetilde{\Gamma}$ and $\overline{\mathrm{co}} \Gamma_{\mathrm{S}}$ coincide on $V, \widetilde{\Gamma}$ is monotone on $V$, any maximal monotone map containing $\Gamma$ coincides with $\widetilde{\Gamma}$ on $V$.

Proof. It results from the assumptions that $V$ is contained in the interior of $C$.

i) Assume for contradiction that $a \in V$ and $a^{*} \in \widetilde{\Gamma}(a)$ exists such that $a^{*} \notin \overline{\mathrm{co}}\left(\Gamma_{\mathrm{S}}(a)\right)$. In view of separation theorems, there exists a vector $d,\|d\|=1$, such that

$$
\sup \left[\left\langle d, \xi^{*}-a^{*}\right\rangle: \xi^{*} \in \operatorname{co}\left(\Gamma_{\mathrm{S}}(a)\right)\right]<0 .
$$

Theorem 2.3 implies that a compact set $K \subset X^{*}$ and an open neighborhood $W \subset V$ of $a$ exist such that $\widetilde{\Gamma}(x) \subset K$ for all $x \in W$. Since $\operatorname{cl}(\mathrm{V} \cap \mathrm{S})=\operatorname{cl}(\mathrm{V})$, there exist a sequence of vectors $\left\{d_{k}\right\} \in X$ converging to $d$ and a sequence of positive real numbers $\left\{t_{k}\right\}$ converging to 0 such that $x_{k}=a+t_{k} d_{k} \in S \cap W$ for all $k$. Since 
$\emptyset \neq \Gamma\left(x_{k}\right) \subset K$ for all $k$ and $K$ is compact, we can find $\bar{x}^{*} \in K$ and a subsequence $\left\{\left(x_{k_{l}}, x_{k_{l}}^{*}\right)\right\} \subset \operatorname{graph}(\Gamma)$ converging to $\left(a, \bar{x}^{*}\right)$. Then $\bar{x}^{*} \in \Gamma_{\mathrm{S}}(a)$.

Next, since $a^{*} \in \widetilde{\Gamma}(a)$ and the subsequence $\left\{\left(x_{k_{l}}, x_{k_{l}}^{*}\right)\right\}$ is contained in graph $(\Gamma)$, one has

$$
\left\langle x_{k_{l}}^{*}-a^{*}, d_{k_{l}}\right\rangle=\frac{1}{t_{k_{l}}}\left\langle x_{k_{l}}^{*}-a^{*}, x_{k_{l}}-a\right\rangle \geq 0 .
$$

Passing to the limit when $k_{l}$ goes to $+\infty$, we obtain $\left\langle\bar{x}^{*}-a^{*}, d\right\rangle \geq 0$, in contradiction with (4). We have proved that $\widetilde{\Gamma}(x) \subset \overline{\mathrm{co}}\left(\Gamma_{\mathrm{S}}(x)\right)$ for all $x \in V$. Since $\overline{\mathrm{co}}\left(\Gamma_{\mathrm{S}}\right)$ is monotone, $\widetilde{\Gamma}$ is monotone on $V$.

ii) Because graph $\left(\Gamma_{\mathrm{S}}\right) \subset \operatorname{cl}(\operatorname{graph}(\Gamma)) \cap\left(\operatorname{cl}(\mathrm{S}) \times \mathrm{X}^{*}\right), \operatorname{cl}(\operatorname{graph}(\Gamma)) \subset \operatorname{graph}(\widetilde{\Gamma})$ and $\operatorname{cl}(\mathrm{V} \cap \mathrm{S})=\operatorname{cl}(\mathrm{V})$, the inclusion $\widetilde{\Gamma}(x) \supset \Gamma_{\mathrm{S}}(x)$ holds for all $x \in V$. Next, since $\widetilde{\Gamma}(x)$ is closed and convex, one has $\widetilde{\Gamma}(x) \supset \overline{\mathrm{co}}\left(\Gamma_{\mathrm{S}}(x)\right)$ for all $x \in V$. In combining with i) one obtains $\widetilde{\Gamma}(x)=\overline{\mathrm{co}}\left(\Gamma_{\mathrm{S}}(x)\right)$

iii) If $\Sigma$ is a maximal monotone map containing $\Gamma$, it contains $\overline{c o}\left(\Gamma_{S}\right)$ and is contained in $\widetilde{\Gamma}$. Hence, it coincides with $\widetilde{\Gamma}$ on $V$.

Proposition 2.7. Let $\Gamma: X \rightrightarrows X^{*}$ be monotone. Assume that $\bar{x} \in \operatorname{bd}(\mathrm{C})$ and there exist $S \subset \operatorname{dom}(\Gamma)$ and an open neighborhood $V$ of $\bar{x}$ such that $\operatorname{cl}(\mathrm{V} \cap \mathrm{S})=\mathrm{cl}(\mathrm{V} \cap \mathrm{C})$. Assume in addition that there exist $\bar{d} \in \operatorname{int}\left(\mathrm{T}_{\mathrm{C}}(\overline{\mathrm{x}})\right)$ and a sequence $\left\{\left(x_{k}, x_{k}^{*}\right)\right\}_{k} \subset\left(S \times X^{*}\right) \cap \operatorname{graph}(\Gamma)$ such that $x_{k}=\bar{x}+t_{k} d_{k}$ for all $k, t_{k} \rightarrow 0_{+}, d_{k} \rightarrow \bar{d}$ and $\left\|x_{k}^{*}\right\| \rightarrow+\infty$ when $k \rightarrow+\infty$. Then, $\Gamma_{\mathrm{S}}(\bar{x})=\widetilde{\Gamma}(\bar{x})=\emptyset$. Moreover, for any sequence $\left\{\left(y_{k}, y_{k}^{*}\right)\right\}_{k} \subset \operatorname{graph}(\Gamma)$ such that the sequence $\left\{y_{k}\right\}_{k}$ converges to $\bar{x},\left\|y_{k}^{*}\right\|$ goes to $+\infty$ when $k$ goes to $+\infty$.

Proof. i) Since $\Gamma_{\mathrm{S}}(\bar{x}) \subset \widetilde{\Gamma}(\bar{x})$, it is enough to show that $\widetilde{\Gamma}(\bar{x})=\emptyset$. Assume, for contradiction, that there is some $\bar{x}^{*} \in \widetilde{\Gamma}(\bar{x})$. Without loss of generality, we assume that the whole sequence $\left\{\frac{x_{k}^{*}}{\left\|x_{k}^{*}\right\|}\right\}$ converges to some $w^{*}$. It results from the assumptions that, for all $k$,

$$
\left\langle\frac{x_{k}^{*}}{\left\|x_{k}^{*}\right\|}-\frac{\bar{x}^{*}}{\left\|x_{k}^{*}\right\|}, d_{k}\right\rangle=\frac{1}{t_{k}\left\|x_{k}^{*}\right\|}\left\langle x_{k}^{*}-\bar{x}^{*}, x_{k}-\bar{x}\right\rangle \geq 0 .
$$

Passing to the limit, one obtains

$$
\left\langle w^{*}, \bar{d}\right\rangle \geq 0
$$

On the other hand, for all $\left(z, z^{*}\right) \in \operatorname{graph}(\widetilde{\Gamma})$ with $z \in \operatorname{int}(\mathrm{C})$ and for all $k$, one has $\left\langle x_{k}^{*}-z^{*}, x_{k}-z\right\rangle \geq 0$. Hence, passing to the limit, one obtains $\left\langle w^{*}, \bar{x}-z\right\rangle \geq 0$. One deduces that $w^{*} \in N_{C}(\bar{x})$. Then $\left\langle w^{*}, \bar{d}\right\rangle<0$ because $\left\|w^{*}\right\|=1$ and $\bar{d} \in \operatorname{int}\left(\mathrm{T}_{\mathrm{C}}(\bar{x})\right)$, in contradiction with (5).

ii) If $\left\|y_{k}^{*}\right\|$ does not go to $+\infty$ when $k$ goes to $+\infty$, then there are $y^{*}$ and a subsequence of $\left\{\left(y_{k}, y_{k}^{*}\right)\right\}_{k}$ converging to $\left(\bar{x}, y^{*}\right)$. Then $\left(\bar{x}, y^{*}\right) \in \operatorname{graph}(\widetilde{\Gamma})$, in contradiction with the first part of the theorem.

Theorem 2.8. Let $\Gamma: X \Longrightarrow X^{*}$ be monotone. Assume that $\bar{x} \in \operatorname{bd}(\mathrm{C})$ and that there exist a subset $\mathrm{S} \subset \operatorname{dom}(\Gamma)$ and an open neighborhood $V$ of $\bar{x}$ satisfying $\operatorname{cl}(\mathrm{V} \cap \mathrm{S})=\mathrm{cl}(\mathrm{V} \cap \mathrm{C})$. Then,

$$
\left.\widetilde{\Gamma}(\bar{x})=\overline{\mathrm{co}}\left(\Gamma_{\mathrm{S}}(\bar{x})\right)\right)+\mathrm{N}_{\mathrm{C}}(\bar{x}) .
$$

Proof. The inclusion $\overline{\mathrm{co}}\left(\Gamma_{\mathrm{S}}(\bar{x})\right)+\mathrm{N}_{\mathrm{C}}(\bar{x}) \subset \widetilde{\Gamma}(\bar{x})$ is a consequence of Propositions 1.2 and 2.1. Let us prove the reverse inclusion. Firstly, we prove that $\overline{\mathrm{co}}\left(\Gamma_{\mathrm{S}}(\bar{x})\right)+\mathrm{N}_{\mathrm{C}}(\bar{x})$ is closed. Since we are faced with the sum of two closed convex sets it is sufficient to prove that the intersection of their recession cones is reduced to the origin. Note that the inclusion $\left.\widetilde{\Gamma}(\bar{x}) \supset \overline{\mathrm{co}}\left(\Gamma_{\mathrm{S}}(\bar{x})\right)\right)$ and Proposition 2.1 imply

$$
\left[\overline{\mathrm{CO}}\left(\Gamma_{\mathrm{S}}(\bar{x})\right)\right]_{\infty} \subset(\widetilde{\Gamma}(\bar{x}))_{\infty}=\mathrm{N}_{\mathrm{C}}(\bar{x}) .
$$

On the other hand, $-N_{C}(\bar{x}) \cap N_{C}(\bar{x})=\{0\}$ because int $(\mathrm{C}) \neq \emptyset$. Therefore

$$
-N_{C}(\bar{x}) \cap\left[\overline{\mathrm{co}}\left(\Gamma_{\mathrm{S}}(\bar{x})\right)\right]_{\infty} \subset-\mathrm{N}_{\mathrm{C}}(\bar{x}) \cap \mathrm{N}_{\mathrm{C}}(\bar{x})=\{0\} .
$$


Next, assume, for contradiction, that there exists $\bar{x}^{*} \in \widetilde{\Gamma}(\bar{x})$ such that $\bar{x}^{*} \notin \overline{\mathrm{co}}\left(\Gamma_{\mathrm{S}}(\bar{x})\right)+\mathrm{N}_{\mathrm{C}}(\bar{x})$. Since $\overline{\mathrm{co}}\left(\Gamma_{\mathrm{S}}(\bar{x})\right)+\mathrm{N}_{\mathrm{C}}(\bar{x})$ is closed and convex, in view of separation theorems, a vector $\bar{d},\|\bar{d}\|=1$, exists such that

$$
\sup \left[\left\langle\bar{d}, x_{1}^{*}+x_{2}^{*}\right\rangle: x_{1}^{*} \in \Gamma_{\mathrm{S}}(\bar{x}), x_{2}^{*} \in N_{C}(\bar{x})\right]<\left\langle\bar{d}, \bar{x}^{*}\right\rangle .
$$

Since $N_{C}(\bar{x})$ is a cone,

$$
0=\sup \left[\left\langle\bar{d}, x_{2}^{*}\right\rangle: x_{2}^{*} \in N_{C}(\bar{x})\right]
$$

which says that $\bar{d} \in T_{C}(\bar{x})$. Next, (6) and (7) imply

$$
\sup \left[\left\langle\bar{d}, x_{1}^{*}-\bar{x}^{*}\right\rangle: x_{1}^{*} \in \Gamma_{\mathrm{S}}(\bar{x})\right]<0 .
$$

Since int $(\mathrm{C})$ is not empty, there exists $d \in \operatorname{int}\left(\mathrm{T}_{\mathrm{C}}(\bar{x})\right)$ such that

$$
\sup \left[\left\langle d, x_{1}^{*}-\bar{x}^{*}\right\rangle: x_{1}^{*} \in \Gamma_{\mathrm{S}}(\bar{x})\right]<0 .
$$

Since $d \in \operatorname{int}\left(\mathrm{T}_{\mathrm{C}}(\bar{x})\right)$, there is a sequence $\left\{\left(x_{k}, x_{k}^{*}\right)\right\} \subset\left(\mathrm{S} \times \mathrm{X}^{*}\right) \cap \operatorname{graph}(\Gamma)$ such that $t_{k} \rightarrow 0_{+}, d_{k} \rightarrow d$ and $x_{k}=\bar{x}+t_{k} d_{k} \in \operatorname{int}(\mathrm{C})$ for all $k$. Since $\widetilde{\Gamma}(\bar{x})$ is not empty, Proposition 2.7 implies that the sequence $\left\{x_{k}^{*}\right\}$ is bounded. Without loss of generality it can be assumed that the whole sequence $\left\{x_{k}^{*}\right\}$ converges to some $x^{*}$. Then $x^{*} \in \Gamma_{\mathrm{S}}(\bar{x}) \subset \widetilde{\Gamma}(\bar{x})$.

Then the definition of $\widetilde{\Gamma}$ implies

$$
\left\langle\bar{x}^{*}-x_{k}^{*}, d_{k}\right\rangle=\frac{1}{t_{k}}\left\langle\bar{x}^{*}-x_{k}^{*}, x_{k}-\bar{x}\right\rangle \leq 0 \text { for all } k .
$$

Passing to the limit, one obtains $\left\langle\bar{x}^{*}-x^{*}, d\right\rangle \leq 0$, in contradiction with (8).

The combination of Theorems 2.6 and 2.8 gives birth to the following result.

Theorem 2.9. Let $\Gamma: X \longrightarrow X^{*}$ be monotone. Assume that the interior of $C=\overline{\operatorname{co}}(\operatorname{dom}(\Gamma))$ is not empty and there exists $\mathrm{S} \subset \operatorname{dom}(\Gamma)$ such that $\mathrm{cl}(\mathrm{S})=\mathrm{C}$. Then, the multi-valued map $\Lambda: X \rightrightarrows X^{*}$ defined by

$$
\Lambda(x)=\left\{\begin{array}{cl}
\overline{\mathrm{co}}\left(\Gamma_{\mathrm{S}}(x)\right)+\mathrm{N}_{\mathrm{C}}(x) & \text { if } x \in C, \\
\emptyset & \text { if } x \notin C,
\end{array}\right.
$$

is the unique maximal monotone map containing $\Gamma$ with domain contained in $C$.

Proof. Let $x \in C$. Then theorems 2.6 and 2.8 imply $\Lambda(x)=\widetilde{\Gamma}(x)$. Hence $\Lambda(x)=\widetilde{\Lambda}(x)$.

It remains to prove that $\widetilde{\Lambda}(x)=\emptyset$ for all $x \notin C$. Assume, for contradiction, that there is $\left(a, a^{*}\right) \in \operatorname{graph}(\Lambda)$ with $a \notin C$. Define $\Sigma: X \longrightarrow X^{*}$ by $\operatorname{graph}(\Sigma)=\operatorname{graph}(\Lambda) \cup\left\{\left(\mathrm{a}, \mathrm{a}^{*}\right)\right\}$. Then $\Sigma$ is monotone.

Let $b \in \operatorname{int}(\mathrm{C})$ and $\bar{t} \in(0,1)$ be such that $c=b+\bar{t}(a-b) \in \operatorname{bd}(\mathrm{C})$. Denote by $D$ the convex hull of $C \cup\{a\}$. Then $c \in \operatorname{int}(\mathrm{D})$. Therefore $\Sigma$ is bounded in a neighbourhood of $c$. If $\Sigma(c)$ is not empty, then $\Sigma(c)=\Lambda(c)+N_{C}(c)$ which is unbounded. If $\Sigma(c)$ is empty, then $\Sigma(b+t(a-b))=\Lambda(b+t(a-b))$ becomes unbounded when $t \rightarrow \bar{t}$ with $t<\bar{t}$. In both cases we have a contradiction.

Thus $\Lambda$ coincides with $\widetilde{\Lambda}$ and therefore is maximal monotone. It coincides with $\widetilde{\Gamma}$ on $C$. We know that $\widetilde{\Gamma}$ contains any monotone map containing $\Gamma$. We deduce that $\Lambda$ is the unique maximal monotone map containing $\Gamma$ with domain contained in $C$.

Remark 2.10. This theorem says that if a monotone map is known on a dense set, it has an unique maximal monotone extension to the closure of the set. As a corollary, we recover the fact that the subdifferential of a convex function can be recovered from the gradients of the function at points where it is differentiable. 


\section{DeAling With Relative interiors}

This short section extends the results of section 3 to the case where the interior of the convex hull of the domain of $\Gamma: X \longrightarrow X^{*}$ is empty. The affine hull of dom $(\Gamma)$ is of the form

$$
\operatorname{aff}(\operatorname{dom}(\Gamma))=\mathrm{a}+\mathrm{X}_{1} \text { where } \mathrm{X}_{1} \text { is a linear subspace of } \mathrm{X} \text { and } \mathrm{a} \in \operatorname{dom}(\Gamma) \text {. }
$$

We denote by $X_{2}$ the orthogonal space to $X_{1}$,

$$
X_{2}=\left\{x^{*} \in X^{*}:\left\langle x^{*}, x\right\rangle=0 \text { for all } x \in X_{1}\right\}
$$

Here again, we distinguish the different roles played by the primal and dual variables by denoting by $X_{1}^{*}$ and $X_{2}^{*}$ the dual spaces of $X_{1}$ and $X_{2}$.

We associate with $\Gamma$ the map $\Sigma: X_{1} \longrightarrow X_{1}^{*}$ defined by

$$
\Sigma\left(x_{1}\right)=\left\{x_{1}^{*} \in X_{1}^{*}: \exists x_{2}^{*} \in X_{2}^{*} \text { with } x_{1}^{*}+x_{2}^{*} \in \Gamma\left(a+x_{1}\right)\right\} .
$$

The proof of the following result is easy, it is left to the reader.

Proposition 3.1. $\Gamma$ is monotone if and only if $\Sigma$ is monotone. $\Gamma$ is maximal monotone if and only if $\Sigma$ is maximal monotone and $\Gamma\left(a+x_{1}\right)=\Sigma\left(x_{1}\right)+X_{2}^{*}$ for all $x_{1} \in X_{1}$.

By the way of this proposition the results of section 3 are easily extended to (maximal) monotone maps $\Gamma$. In particular we straightforwardly recover the following results.

Proposition 3.2. Let $\Gamma: X \longrightarrow X^{*}$ be maximal monotone. Then the relative interior and the closure of its domain are convex. Moreover the closure of the domain coincides with the closure of its relative interior.

Proposition 3.3. Let $\Gamma: X \longrightarrow X^{*}$ be monotone. Denote by $C$ the closed convex hull of its domain. Then, for all $\bar{x} \in \mathrm{ri}(\mathrm{C})$, there is a neighbourhood $V$ of $\bar{x}$ and a compact set $K \subset X_{1}^{*}$ such that for all $x \in V \cap C$ it holds

$$
\emptyset \neq \widetilde{\Gamma}(x)=\widetilde{\Gamma}(x)+X_{2}^{*} \subset K+X_{2}^{*}
$$

\section{A construction of a maximal monotone extension}

In this section, we show how to construct a maximal monotone extension of a monotone map $\Gamma: X \rightleftarrows X^{*}$ (or equivalently a maximal extension of its graph $G$ ). Unlike the traditional proof of the existence of such an extension which is based on the axiom of choice, we give a constructive proof based on the density properties seen in Section 3.

Of course, if a subset $G$ of $X \times X^{*}$ is not monotone, it has no maximal monotone extension. In the other case, the extension is not unique as seen from the following example.

Example 4.1. The set $G=\{(0,0),(0,1)\} \subset \mathbb{R}^{2}$. The sets $G_{1}=[(-\infty, 0] \times\{0\}] \cup[\{0\} \times[0,1] \cup[0, \infty) \times\{1\}]$ and $G_{2}=\{0\} \times \mathbb{R}$ are two maximal monotone extensions of $G$.

In our construction we have chosen to work on $\Gamma$, but the same construction can be done on $\Gamma^{-}$. One gets different maximal monotone extensions.

Let $\Gamma: X \longrightarrow X^{*}$ be monotone, let $C$ be the closure of the convex hull of its domain. Assume first that the interior of $C$ is not empty. Consider some countable set $S=\left\{x_{0}, x_{1}, \cdots, x_{k}, \cdots\right\} \subset$ int $(\mathrm{C})$ such that $\mathrm{cl}(\mathrm{S})=\mathrm{C}$; such sets $S$ exist. The first part of the construction is described in the following algorithm.

Algorithm: 
- Step 0: Define $\Gamma_{0}: X \Longrightarrow X^{*}$ as

$$
\Gamma_{0}(x)=\left\{\begin{array}{cc}
\Gamma(x)+N_{C}(x) & \text { if } x \in C, \\
\emptyset & \text { if not }
\end{array}\right.
$$

By construction, $\Gamma_{0}$ is monotone and $\operatorname{dom}\left(\Gamma_{0}\right)=\operatorname{dom}(\Gamma)$.

Step k: In the previous step a monotone map $\Gamma_{k}$ has been obtained with graph $\left(\Gamma_{\mathrm{k}}\right) \supset \operatorname{graph}(\Gamma)$.

- If $\operatorname{dom}\left(\Gamma_{\mathrm{k}}\right) \supset \mathrm{S}$, the multi-valued map $\Lambda: x \Longrightarrow \widetilde{\Gamma}_{k}(x)+N_{C}(x)$ is maximal monotone (Theorem 2.9). STOP.

- Otherwise, take

$$
p(k)=\min \left[p \in \mathbb{N}: x_{p} \notin \operatorname{dom}\left(\Gamma_{\mathrm{k}}\right)\right],
$$

and define a new map $\Gamma_{k+1}$ as follows

$$
\Gamma_{k+1}(x)=\left\{\begin{array}{cl}
\Gamma_{k}(x) & \text { if } x \in \operatorname{dom}\left(\Gamma_{\mathrm{k}}\right), \\
\widetilde{\Gamma}_{k}\left(x_{p(k)}\right) & \text { if } x=x_{p(k)}, \\
\emptyset & \text { otherwise }
\end{array}\right.
$$

By construction, $\Gamma_{k+1}$ is monotone,

$$
\begin{aligned}
& \operatorname{dom}\left(\Gamma_{\mathrm{k}+1}\right)=\operatorname{dom}\left(\Gamma_{\mathrm{k}}\right) \cup\left\{\mathrm{x}_{\mathrm{p}(\mathrm{k})}\right\} \subset \mathrm{C}, \\
& \operatorname{graph}(\Gamma) \subset \operatorname{graph}\left(\Gamma_{\mathrm{k}}\right) \subset \operatorname{graph}\left(\Gamma_{\mathrm{k}+1}\right),
\end{aligned}
$$

and $p(k+1) \geq p(k)+1$.

Do $k=k+1$ and go back to step $k$.

\section{End of the algorithm.}

In the second part of the construction, we consider $D=\cup_{k} \operatorname{dom}\left(\Gamma_{\mathrm{k}}\right)$. Since $S \subset D \subset C$ one has $\operatorname{cl}(\mathrm{D})=\mathrm{C}$. Next, define $\Lambda: X \Longrightarrow X^{*}$ by

$$
\Lambda(x)=\left\{\begin{array}{cl}
\Gamma_{k}(x) & \text { if } x \in \operatorname{dom}\left(\Gamma_{\mathrm{k}}\right) \\
\emptyset & \text { if } x \notin D
\end{array}\right.
$$

By construction, $\Lambda$ is monotone and $C=\operatorname{cl}(\operatorname{dom}(\Lambda))$. Next, define $\Omega: X \Longrightarrow X^{*}$ by $\Omega(x)=\widetilde{\Lambda}(x)+N_{C}(x)$ for all $x$. It follows from Theorem 2.9 that $\Omega$ is maximal monotone, contains $\Gamma$ and $C$ is the closure of its domain.

Other maximal monotone extensions are obtained by considering another $S$, or in working with $\Gamma^{-}$instead of $\Gamma$. They do not coincide in general.

In the case where the interior of $C$ is empty, a maximal monotone extension of $\Gamma$ is obtained in constructing a maximal monotone extension $\Phi$ of the map $\Sigma: X_{1} \rightrightarrows X_{1}^{*}$ defined in section 4 , next in taking $\Omega$ such that $\Omega\left(a+x_{1}\right)=\Phi\left(x_{1}\right)+X_{2}^{*}$ for all $x_{1} \in X_{1}$. Here again, $\Omega$ is maximal monotone, contains $\Gamma$ and $C$ is the closure of its domain.

\section{REFERENCES}

[1] A. Auslender and M. Teboulle, Asymptotic cones and functions in optimization and variational inequalities, Springer-Verlag, New York Inc., 2003.

[2] H. Brezis, Opérateurs Maximaux Monotones et Semigroupes de Contractions dans les Espaces de Hilbert, North Holland, Amsterdam, 1973.

[3] F. Facchinei and J.S. Pang, Finite dimensional variational inequalities and complementarity problems, Vol.1 and Vol.2, Springer-Verlag, New York Inc., 2003.

[4] R.I. Kachurovskii, On monotone operators and convex functionals, Uspekhi Matematicheskikh Nauk 15, 1960, $213-215$.

[5] R.I. Kachurovskii, Nonlinear monotone operators in Banach spaces, Uspekhi Matematicheskikh Nauk 23, 1968, 121-168.

[6] C. Lescarret, Cas d'addition des applications maximales dans un espace de Hilbert, C. R. Acad. Sci. Paris, 261, 1965, 11601163. 
[7] J.-E. Martinez-Legaz and B.F. Svaiter, Monotone Operators Representable by l.s.c. Convex Functions, Set-Valued Analysis, $13,2005,21-46$.

[8] G.J. Minty, Monotone Networks, Proceeding of the Royal Society of London (Serie A) 257, 1960, 194-212.

[9] G.J. Minty, On the maximal domain of a monotone function, Michigan Mathematical Journal, 8, 1961, $135-137$.

[10] G.J. Minty, Monotone (nonlinear) operators in Hilbert Space, Duke Mathematics Journal 29, 1962, 341-346.

[11] R. R. Phelps, Convex functions, Monotone Operators, and Differentiability, Lecture notes in mathematics, 1364, Springer, New York, 1989

[12] R.T. Rockafellar, Convex Analysis, Princeton University Press, Princeton, New Jersey, 1970.

[13] R.T. Rockafellar, On the maximality of sums of nonlinear monotone operators, Trans. Amer. Math. Soc., 149, 1970, 75-88.

[14] R.T. Rockafellar, On the virtual convexity of the domain and range of a nonlinear maximal monotone operator, Mathematische Annalen, 185, 1970, 81-90.

[15] R.T. Rockafellar, Monotone operators and the proximal point algorithm, SIAM J. Control Optim. 14, 1976, 877-898.

[16] R.T. Rockafellar and R.J.B Wets, Variational Analysis, Springer-Verlag, New York, 1998.

[17] E.H. Zarantonello, La structure des opérateurs monotones, Rev. Un. Mat. Argentina 34, 1990, 90-96. 